\title{
Two adolescents with gluten sensitivity, non celiac, not wheat allergy, associated with non specific inflammatory bowel disease
}

Keywords: wheat, barley, rye, celiac disease, wheat allergy, non celiac gluten sensitivity, inflammatory bowel disease, gluten free diet

Abbreviations: CD, celiac disease; WA, wheat allergy; NCGS, non celiac gluten sensitivity; IBD, inflammatory bowel disease; GFD, gluten free diet

\section{Aim}

Describe clinic, endoscopic and histopathology findings, and response to treatment in a 12 years old boy and a 17 year old girl, With NCGS, associated to IBD.

\section{Background}

$\mathrm{CD}$ is an autoimmune systemic disorder, triggered by gluten and related prolamines present in wheat, barley and rye, that occur in genetically susceptible individuals, who have the human leukocyte antigen HLA-DQ2 and/or HLA-DW8 haplotypes. It is caracterized by inflammatory enteropathy, with variable degrees of severity, a wide range of gastrointestinal and/or systemic complaints, and the presence of celiac-specific auto-anti-bodies; is the more frequent inflammatory gastrointestinal disease in west countries. ${ }^{1-4}$

We describe two adolescents with digestive and systemic symptoms and signs related to gluten ingestion, with abnormal histopathologic findings and abscence of gluten and transaminase auto-antibodies, $\operatorname{IgG}$ and $\operatorname{IgA}$, and negative for wheat IgE allergy, they had clinical remission on gluten free diet, and exaceration when they eat gluten, both associated to non-specific IBD, wich improved with prednisone.

a. What is known

i. CD can start at any age.

ii. The signs and symptoms vary widely.

iii. The difference between children and adults is that; children, could have failure to thrive and short stature.

iv. Both can have both gastro-intestinal and extra-intestinal manifestations.

v. Extra-intestinal manifestations of $\mathrm{CD}$ can improved o disappeared with gluten free diet, as in the boy, in which chronic constipation, since pre-school age to up tu date, finished in few days, when he started free gluten diet.

b. What is less known

i. Chronic constipation could be the only symptom of CD.

ii. IBD can be associated to CD.

c. What is new

i. IBD can be associated, not only to CD, but to NCGS, and, perhaps, WA.
Volume 7 Issue 4 - 2017

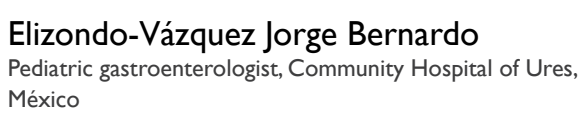
México

Correspondence: Elizondo-Vázquez Jorge Bernardo, Pediatric gastroenterologist. Community Hospital of Ures, Sonora, Health Secretary of Sonora State, México, Email jelizondovazquez@gmail.com

Received: June 21, 2017 | Published: August 18, 2017

\section{Case report}

\section{Case I}

A 12 years old boy begin with anorexia and weight loss (49 to 40.8 $\mathrm{Kg}$ ) since january to april12-17. He has normal activity.

Since baby, his bowel movements were 1-2 per week, Bristol 1 to 3 , feces frequently stuck in toilet.

Exploration: Weight $40.8 \mathrm{Kg}$, height 1.58meters. BMI 16.3 (C 25). Thin, active, flat abdomen, without active neither passive pain, nor abnormal bowel sounds.

Laboratory: Negative anti-gliadin IgG and IgA, anti-transglutaminase IgA, anti-total and wheat IgE.

High Endoscopy: Diffuse capillary congestion in stomach. Duodenum is, macroscopically, normal.

Coloncoscopy: Normal ileum. Deformed cecal valve by edema. Ceccum with visible taenias. Capillaries are lost in surrounding appendiceal mucosa. Mucosa, since ceccum to two proximal thirds of transverse colon, with paralel folds, formed by diffuse edema, and diffuse punctate hemorrhage.

Distal transversal and descending colon with normal mucosa. Capillaris are lost in sigmoid by edema, diffuse hemorrhage and whitsh, round, and tiny superficial injuries.

\section{Pathology}

a. Stomach: Well conserved mucosa; edema, capillar congestion and mild-moderate chronic inflammation of lamina propia. No Helicobacter pylori was found. 
b. Duodenum: Digital villi with cilindric epithelium, integral brush border. Mild infiltration with lymphocites and plasma cells (Marsh 1).

c. Ileum: Mucosa with nodular chronic inflammatory infiltrate, with mild shortness of villi (Peyer Patch).

d. Colon: Mild crypt desorganization; epitheliun with goblet cells. Some fragments of lamina propia are congestive, with edema; others, with fibrosis. Some little lymphoid aggregates are identifided.

e. Sigmoid colon: Fibrosis and mild to moderate chronic inflammation in lamina propia. Mild crypt distortion.

\section{Case 2}

A 17 years old girl, she begin at 13 years old with bloating and abdominal pain, loose stools and facial and leg edema, she noticed symptoms were worse when she eat wheat; she almost left wheat out from her diet, her symptoms improved; but, almost all time, has facial and leg edema, and abdominal malaise; the symptoms and signs exacerbated when she increased gluten ingestion; she was instructed to eat daily gluten foods, for 15 days, before blood extraction, for celiac antibodies. At day three, facial edema, bloating and abdominal pain worse; she have to stop wheat from her diet, anto-antibodiies were taken at that time.

Exploration: Weight $66 \mathrm{Kg}$, height $1.65 \mathrm{~m}$. Abdominal circumference $98 \mathrm{~cm}$, soft, diffuse pain on palpation, audible and palpable borborigm, continouos and high intensity peristalsis.

Laboratory: Anti-deaminated gliadin $\operatorname{IgG}$ and $\operatorname{IgA}$, $\operatorname{IgA}$ antitransglutaminase, total $\operatorname{IgE}$ and anti-wheat $\operatorname{IgE}$ are negative.

Endoscopy: Erosive gastritis, normal macroscopic mucosa in duodenum. Diffuse colitis with erithema, edema and patchy and diffuse hemorrhage.

Pathology: Stomach: Erosive gastritis.

Duodenum: Villi height diminished 50\%, lamina propia with intense infiltratration with lymphocites and plasma cells, MARSH 2.

Colon: Epithelium is infiltrated with lymphocites; goblet cells without mucus, and without luminal inflammatory cell infiltration. Lamina propia has severe infiltration with mononuclear cells.

\section{Follow Up}

Both cases improved with gluten free diet and prednisone, $1 \mathrm{mg}$ / $\mathrm{Kg}$ /day in one dose, which were diminished, progressivly, in eigth to 12 weeks, until it was withdrawn. The boy reached $48 \mathrm{Kg}$ in less than two months (BMI 19.2C 75), his bowel movements change; since, one to three per week, Bristol 1 to 3, with excessive effort and pain, and one hour attempts in toilet; to one to three per day; Bristol 4 , without effort neither pain, and less than five to ten minutes in toilet. She impoved her abdominal circunference in two weeks, from 97 to $77 \mathrm{~cm}$, facial and leg edema disappeared. She referred less abdominal pain.

\section{Discussion}

$\mathrm{CD}$ is associated to other disorders as autoinmune diseases (herpetic dermatitis, type 1 diabetes, tiroiditis, selective deficit of IgA, IBD, Sjogren syndrome, lupus erythmatosus, Addison disease, IgA nephropathy, chronic hepatitis, primary biliary cirrhosis, reumatoid arthritis, psoriasis, vitiligo and alopecia areata. ${ }^{5}$
WA is a hyper-sensitivity reaction to wheat proteins mediated through immune mechanisms, and involving mast cell activation. The immune response can be immunoglubulin E (IgE) mediated, non-IgE mediated, or a combination of both. WA is most commonly a food allergy, but, wheat can become a sensitizer when the exposition occurs through the skin or airways (baker's asthma). ${ }^{1}$ NCGS is a poorly defined syndorme, characterized by a variable combination of intestinal and extraintestinal symptoms, typically occurring soon after ingestión of gluten-containing food, and disappearing quickly upon their withdrawal, occurring in individuals where, both, CD and WA have been excluded. ${ }^{6}$

Extraintestinal Manifestations of Celiac Disease dissapeared with gluten free diet. ${ }^{7}$ This two adolescents have signs and symptoms with gluten ingestion, not related to $\mathrm{CD}$, neither WA, but are associated to non specific IBD, both improved with gluten free diet and prednisone.

He has a short time with one symptom (anorexia) and one sign (lost weight) in less than four months; he recovered in less than two months, probably related to IBD. The other symptom, chronic constipation, was related to guten ingestión, it disappeared, in less than a week, after years of chronic constipation, without laxatives, only with gluten free diet. She has a long lasting follow up with bloating and abdominal pain, facial and legs edema; all of them improved; The surprising thing was the rapid diminution of the abdominal perimeter, after two weeks, with gluten free diet and prednisone.

The boy changed his bowel movements with gluten free diet; the findings in lamina propia: edema, congestion and fibrosis are not explained by $\mathrm{CD}$; fibrosis is a not specific finding of IBD; but, in this case; he had not another disease related with fibrosis in lamina propia as: radiation, quimiotherpay, sarcoidosis, amyloid disease, neither graft against host disease, which can cause chronic inflammation and fibrosis. Therefore, the only explanation for fibrosis found in lamina propria is related to inflammatory bowel disease (IBD), in particular, Crohn's disease, which can produce fibrosis, since mucosa through serosa; and not to ulcerative colitis because; the latter one, does not affect lamina propria and does not produce fibrosis.

\section{The sum of Clinical findings}

He: Chronic constipation, anorexia and wheight loss $(8.2 \mathrm{Kg}$ in less than four months).

She: Chronic abdominal pain, bloating, facial and legs edema since 13 years old.

Both: Negative serology for CD, WA and IBD.

\section{Endoscopy high and lower}

a. He: Macroscopically: Normal esophagus, stomach, duoedenum and ileum. Ceccum deformed by edema, with edema and stony mucosa, lose of capillaries and superficial puntacte hemorrhage. Ascending colon and two proximal thirds of transverse colon with mucosa as ceccum, except that they do not have stony mucosa. Normal distal third transverse and descendent colon.

Sigmoid and rectum same as ascending and two proximal thirds of transverse colon, plus, little withish injuries of mucosa.

b. She: Submucous visible vessels on fundus. Normal mucosa in esophagus, stomach and duodenum. Normal ileum mucosa. Cecal valve with edema and erithema. Ceccum with edema and puntactate hemorrahage of mucosa. Ascending transverse colon, sigmoid and rectum with diffuse severe edema, which formed 
parallel folds on mucosa, with lost capillaries, and pathcy, diffuse, non confluent superficial hemorrhage. Normal descending colon.

\section{Pathology}

a. He: Non specific gastropahty, Marsh 1 duodenitis; pathchy diffuse colitis, since ceccum to two proximal thirds of transverse colon; normal distal transverse and descending colon, and diffuse proctitis, with "small erosions" represented by fibosis in lamina propia.

His bowel movements changed, constipation disappeared in less than one week. In his whole life, he has thought Bristol one, two and three, with excesive effort and abdominal and anal pain were normal.

b. She: Erosive gastritis, MARSH 2 in duodenum, and severe chronic inflammation with complete loss of mucus production by golbet cells in colon and sigmoid. He recovered $7.2 \mathrm{Kg}$ in less than two months. She diminished abdominal distention, since 98 to $77 \mathrm{~cm}$ and disappearance of facial and legs edema in two weeks.

These findings could not be explain only by $\mathrm{CD}$, WA neither NCGS; neither, by pathology findings, the diagnosis of IBD can be done; but, with combination of all these findings, we can conclude, they are secondary to another disease related to CD and IBD; perhaps, only is needed, is time to can correctly establish the excact diagnosis; but, at this moment; in both patients, signs and symptoms related to gluten food ingestion and non specific findings of IBD, responded to gluten free diet and prednisone; the final diagnosis is NCGS associated to non-specific IBD, probably Crohn's disease.

\section{Conclusion}

A. Finally, with these two cases, the conclusions are

a. Symptoms and signs of CD and IBD can start since first years of life, or in adolescence.

b. The symptomatology may disappear, within few days to weeks, after starting treatment, whether acute or chronic symptoms or signs.

c. In both cases, the main findings, in endoscopy are in colon.

d. Although normal duodenum mucosa in endoscopy in the girl, she had Marsh 2B changes in pathology.

e. The new knowlege is.

i. GSNC and IBD can coexist.

ii. Fibrosis could be a patognomonic finding, when others pathologies, which can produce fibrosis, have been discarded; in this case, it could be Crohn's disease; nevertheless, it has to do much work in this area to confirm or discard this hypothesis.
B. In routine clinical practice, clinicians

1. Should think in these two groups of diseases, in one hand: $C D$, WA and GSNC disease; and, in the other, IBD, the two more frecuent fenotypes: Ulcerative colitis and Crohn's disease.

2. $\mathrm{CD}, \mathrm{WA}$ and GSNC are diseases with gastro-intestinal and extra-intestinal manifestations [7], reason why its diagnosis is not easy; always, the clinician, must have high suspicion of this group of diseases, to be able to make the diagnosis.

3. The same happens with IBD, whose manifestations are diverse, not specific and; in some cases, may start with extraintestinal symptoms and; as in the other group of diseases, the high rate of diagnostic suspicion is what causes, the physician, can make the diagnosis.

\section{Acknowledgements}

None.

\section{Conflicts of Interest}

There is no conflict of interest.

\section{Funding}

None.

\section{References}

1. Hill ID, Fasano A, Guandalini S, et al. NASPGHAN Clinical Report on the Diagnosis and Treatment of Gluten-related Disorders. JPGN 2016;63(1):156-165.

2. Husby S, Koletzko S, Korponay- Szabó IR, et al. European Society for Pediatric Gastroenterology, Hepatology, and Nutrition guidelines for the diagnosis of coeliac diasease. $J$ Pediatr Gastroenterolo Nutr. 2012;54(1):136-160.

3. Revised criteria for diagnosis of coeliac disease report of Working Group of European Society of Paediatric Gastoenterology and Nutrition. Arch Dis Chil. 65(8):909-911.

4. Green PH, Jabri B. Coeliac disease. Lance. 2003;362:383-391.

5. P Pavón Relinchón, MD García Novo, et al. En Tratado de Gastroenterología, Hepatología y Nutrición Pediátrica de la SEGHNP. 2016:285-291.

6. Sapone A, Bai JC, Ciacci C, et al. Spectrum of gluten-related disorders: consensus on new nomenclature and classification. BMC Med. 2012;10: 13.

7. Jericho H, Sansota N, Guandalini S. Extraintestinal Manifestations of Celiac Disease: Effectiveness of Gluten- Free Diet. J Pediatr Gastroenterolo Nutr. 2017;65(1):75-79. 